\title{
On application of semi-Markov processes superposition to energy systems modeling
}

\author{
Yuriy E. Obzherin ${ }^{1,}$, Stanislav M. Sidorov ${ }^{1}$, and Mikhail M. Nikitin ${ }^{1}$ \\ ${ }^{1}$ Sevastopol State University, Higher Mathematics Chair, 299053 Sevastopol, Russian Federation
}

\begin{abstract}
One of the important tasks of the theory of reliability and efficiency of energy systems is the task of creating information systems for managing energy systems and the transition to intelligent management and engineering. The solution to this problem is possible based on the construction of mathematical models relating to various aspects of the structure and functioning of these systems. The paper, using the example of a superposition of independent semi-Markov processes constructed in the works of V.S. Korolyuk, A.F. Turbin, examines the possibilities of using semi-Markov processes with a common phase state space for modeling energy systems; an illustrative example of the application of this approach is given.
\end{abstract}

\section{Introduction}

Semi-Markov processes (SMP) are widely used to build models and analyze systems for various purposes: technical, industrial, energy, information, economic, biological, etc.

The leading world publishing houses Elsevier, Springer, World Scientific have published a number of monographs on the subject in recent years. [1-5].

Semi-Markov processes can be effectively used to build models relating to various aspects of the structure and functioning of energy systems: reliability, efficiency, monitoring, diagnostics, maintenance and forecasting.

In this paper, by the example of a superposition of independent semi-Markov processes constructed in the works of V.S. Korolyuk, A.F. Turbin and their apprentices $[6,7]$, the possibility of using semi-Markov processes with a common phase state space for energy systems modeling is considered.

\section{Description of independent semi- Markov processes superposition}

Let us consider the semi-Markov model of independent SMPs superposition, following to [6,7].

The system consists of $N$ independent elements (subsystems), functioning of each of which is described by SMP $\xi^{(i)}(\mathrm{t})$ with arbitrary phase space of states $Z^{(i)}$, $i=\overline{1, N}$.

For each $Z^{(i)}$ the decomposition $Z^{(i)}=Z_{1}^{(i)} \cup Z_{0}^{(i)}$, $Z_{1}^{(i)} \cap Z_{0}^{(i)} \neq \varnothing, \quad i=\overline{1, N}$, takes place where $Z^{(i)}$ is interpreting as the set of $i$-th element's(subsystem's) up states, $Z_{0}^{(i)}$ is the set of $i$-th element's(subsystem's) down states.

Let

$$
\begin{gathered}
\varsigma_{(i)}(t)=\chi_{Z_{1}^{(i)}}\left(\xi^{(i)}(t)\right)=\left\{\begin{array}{l}
1, \text { if } \xi^{(i)}(t) \in Z_{1}^{(i)}, \\
0, \text { if } \xi^{(i)}(t) \in Z_{0}^{(i)},
\end{array}\right. \\
\varsigma(\mathrm{t})=\left\{\varsigma_{1}(\mathrm{t}), \varsigma_{2}(\mathrm{t}), \ldots, \varsigma_{\mathrm{N}}(\mathrm{t})\right\} .
\end{gathered}
$$

Vector process $\zeta(t)$ characterize the system's states at the instant $t$ by its element's (subsystem's) up or down states. The set of possible $\zeta(t)$ process values is the set of binary vectors $\bar{d}=\left(d_{1}, d_{2}, \ldots, d_{N}\right)$ from $(00 \ldots 0)$ to $(11 \ldots 1)$, define it as $D=\{\bar{d}\}$.

Let us consider the concept of system failure: on the set of values of binary vectors $D$, a function $g(\bar{d})$ is defined such that:

$$
g(\bar{d})=\left\{\begin{array}{l}
1, \text { if the system is operational with this } \\
\text { combination of its elements state, } \\
0, \text { otherwise. }
\end{array}\right.
$$

Let us define the set of values of the vector $\bar{d}$, at which the system is operational, by $D_{1}$, and the set of values at which the system is inoperable, by $D_{0}$, i.e. $D_{1}=\{\bar{d}: g(\bar{d})=1\}, D_{0}=\{\bar{d}: g(\bar{d})=0\}$, by assumption, $D=D_{1} \cup D_{0}, D_{1} \cap D_{0} \neq \varnothing$.

In $[6,7]$ a superposition of independent processes ПМП $\xi^{(i)}(t), i=\overline{1, N}$ was constructed, and it was shown that the average stationary time to failure $T_{1}$, the average stationary renewal time $T_{0}$ and the stationary availability factor $K_{a}$ can be determined by the following formulas:

\footnotetext{
* Corresponding author: objsev@mail.ru
} 


$$
\begin{gathered}
T_{1}=\sum_{\bar{d} \in D_{1}} \prod_{i=1}^{N} T_{d_{i}}^{(i)}\left\{\sum_{\bar{d} \in D_{1}^{\prime}} \prod_{i=1}^{N} T_{d_{i}}^{(i)} \sum_{j \in G(\bar{d})} \frac{1}{T_{1}^{(j)}}\right\}^{-1}= \\
=\sum_{\bar{d} \in D_{1}} \prod_{i=1}^{N} T_{d_{i}}^{(i)}\left\{\sum_{\bar{d} \in D_{0}^{\prime}} \prod_{i=1}^{N} T_{d_{i}}^{(i)} \sum_{j \in I(\bar{d})} \frac{1}{T_{0}^{(j)}}\right\}^{-1}, \quad(1) \\
T_{0}=\sum_{\bar{d} \in D_{0}} \prod_{i=1}^{N} T_{d_{i}}^{(i)}\left\{\sum_{\bar{d} \in D_{1}^{\prime}} \prod_{i=1}^{N} T_{d_{i}}^{(i)} \sum_{j \in G(\bar{d})} \frac{1}{T_{1}^{(j)}}\right\}^{-1}= \\
=\sum_{\bar{d} \in D_{0}} \prod_{i=1}^{N} T_{d_{i}}^{(i)}\left\{\sum_{\bar{d} \in D_{0}^{\prime}} \prod_{i=1}^{N} T_{d_{i}}^{(i)} \sum_{j \in l(\bar{d})} \frac{1}{T_{0}^{(j)}}\right\}^{-1}, \quad(2) \\
K_{a}=\sum_{\bar{d} \in D_{1}} \prod_{i=1}^{N} T_{d_{i}}^{(i)}\left\{\prod_{i=1}^{N}\left(T_{1}^{(i)}+T_{0}^{(i)}\right)\right\}^{-1},
\end{gathered}
$$

where $T_{1}^{(i)}$ is the average stationary time to failure of the $i$-th element (subsystem); $T_{0}^{(i)}$ is the average stationary renewal time of the $i$-th element (subsystem);

$$
T_{d_{i}}^{(i)}=\left\{\begin{array}{l}
T_{1}^{(i)}, \text { if } d_{i}=1, \\
T_{0}^{(i)}, \text { if } d_{i}=0 ;
\end{array}\right.
$$

$D_{1}^{\prime}$ is the set of vectors $\bar{d} \in D_{1}$ such that changing the value of some single component from one to zero brings the vector $\bar{d}$ into the set $D_{0}\left(D_{1}^{\prime}\right.$ is the set of boundary operational states of the system);

$D_{0}^{\prime}$ is the set of vectors $\bar{d} \in D_{0}$ such that changing the value of some single component from zero to one brings the vector $\bar{d}$ into the set $D_{1}\left(D_{1}^{\prime}\right.$ is the set of boundary system failure states);

$G(\bar{d})$ is the set of vector $\bar{d} \in D_{1}^{\prime}$ component numbers, changing the value of each of which from unit to zero brings the vector $\bar{d}$ into the set $D_{0}$;

$I(\bar{d})$ is the set of vector $\bar{d} \in D_{0}^{\prime}$ component numbers, changing the value of each of which from zero to unit brings the vector $\bar{d}$ into the set $D_{1}$.

The paper [7] shows that the formulae (1) - (3) can be used for immediate calculation of reliability indicators of various typical structures and also, using these formulas and step-by step structure merging algorithm [7], for reliability characteristics estimating for the systems with high number of elements.

Using the formulae (1) - (3) one can also take into account the presence of unloaded reserve, inspection, maintenance, availability of time reserve, etc.

To do this one should find the appropriate expressions for $T_{1}, T_{0}$ of subsystems elements, e.g. from $[8-11]$ and use them in the formulae (1) - (3).
The paper [7] represents approximate formulae for $T_{1}, T_{0}, K_{a}$, valid under the condition of system's elements (subsystems) rapid recovery. Using the semi-Markov superposition model built in $[6,7]$ and the embedded Markov chain stationary distribution found there one can also find other characteristics of the system including that determine the effectiveness of system's operation.

\section{Example of electric energy system modeling}

As an example to illustrate the application of the formulae (1) - (3) to model the electric energy systems let us determine the reliability characteristics of the

\begin{tabular}{|c|c|c|c|c|}
\hline № & Element & $T_{0}, \mathrm{yr}$. & $T_{1}, \mathrm{yr}$. & $K_{a}$ \\
\hline 1 & $\begin{array}{l}\text { Electric power } \\
\text { line }\end{array}$ & 3.0 & 0.04 & 0.9868 \\
\hline 2 & $\begin{array}{l}\text { Substation A } \\
\text { input bus }\end{array}$ & 1.5 & 0.031 & 0.9797 \\
\hline 3 & $\begin{array}{l}\text { Substation A } \\
\text { transformer } 1\end{array}$ & 1.7 & 0.027 & 0.9843 \\
\hline 4 & $\begin{array}{l}\text { Substation A } \\
\text { transformer } 2\end{array}$ & 0.9 & 0.033 & 0.9646 \\
\hline 5 & $\begin{array}{l}\text { Substation B } \\
\text { input bus }\end{array}$ & 2.1 & 0.02 & 0.9906 \\
\hline 6 & $\begin{array}{l}\text { Substation B } \\
\text { transformer } 1\end{array}$ & 1.1 & 0.018 & 0.9839 \\
\hline 7 & $\begin{array}{l}\text { Substation B } \\
\text { transformer } 2\end{array}$ & 1.5 & 0.01 & 0.9934 \\
\hline 8 & $\begin{array}{l}\text { Substation A } \\
\text { output bus }\end{array}$ & 1.4 & 0.014 & 0.9901 \\
\hline 9 & $\begin{array}{l}\text { Substation B } \\
\text { output bus }\end{array}$ & 1.3 & 0.017 & 0.9871 \\
\hline 10 & Switch & 0.5 & 0.037 & 0.9311 \\
\hline 11 & Switch & 0.36 & 0.016 & 0.9574 \\
\hline 12 & Switch & 0.6 & 0.025 & 0.9600 \\
\hline 13 & Switch & 0.8 & 0.015 & 0.9816 \\
\hline 14 & $\begin{array}{l}\text { Electric power } \\
\text { line }\end{array}$ & 1.2 & 0.0014 & 0.9988 \\
\hline 15 & $\begin{array}{l}\text { Electric power } \\
\text { line }\end{array}$ & 2.1 & 0.0015 & 0.9993 \\
\hline
\end{tabular}
electric energy system with the structural diagram represented in the Figure 1 and the elements reliability characteristics represented in the Table 1.

Table 1. Reliability characteristics of the electric energy system's elements 


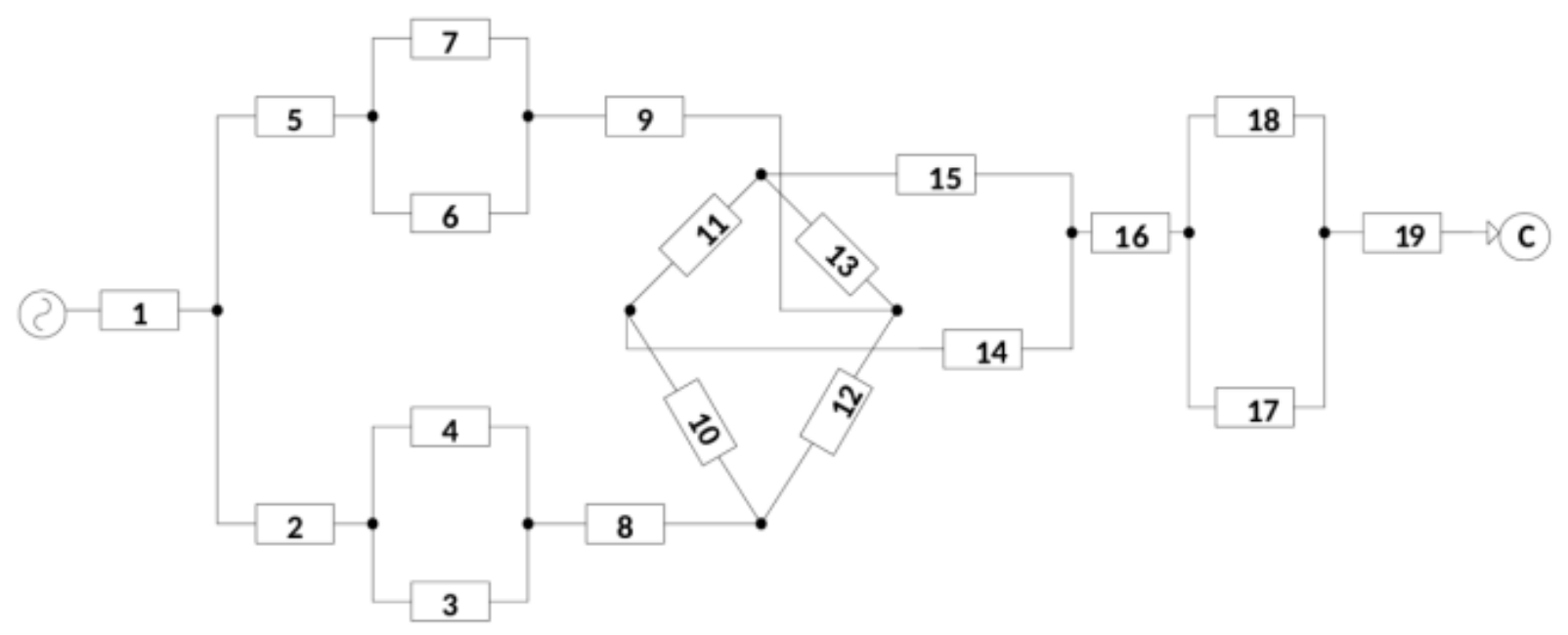

Fig. 1. Structural diagram of the electric energy system.

Table. 2 Reliability characteristics of the electric energy system's elements (continuation)

\begin{tabular}{|c|l|l|l|l|}
\hline 16 & $\begin{array}{l}\text { Substation C } \\
\text { input bus }\end{array}$ & 0.5 & 0.028 & 0.9470 \\
\hline 17 & $\begin{array}{l}\text { Substation C } \\
\text { transformer 1 }\end{array}$ & 1.0 & 0.021 & 0.9794 \\
\hline 18 & $\begin{array}{l}\text { Substation C } \\
\text { transformer 2 }\end{array}$ & 1.1 & 0.024 & 0.9786 \\
\hline 19 & $\begin{array}{l}\text { Substation C } \\
\text { output bus }\end{array}$ & 2.0 & 0.009 & 0.9955 \\
\hline
\end{tabular}

Finding the characteristics of the system considered with the aid help of the formulae (1) - (3) is carried out according to the following scheme:

1. Parallel elements 3, 4, and also 6, 7 are merged to one element.

2. Sequential elements 2,8 and the result of the merging of 3 and 4, as well as 5, 9 and the result of 6 and 7 merging are merged to one element.

As a result, we obtain a merged subsystem, the block diagram of which is presented in Fig. 2.

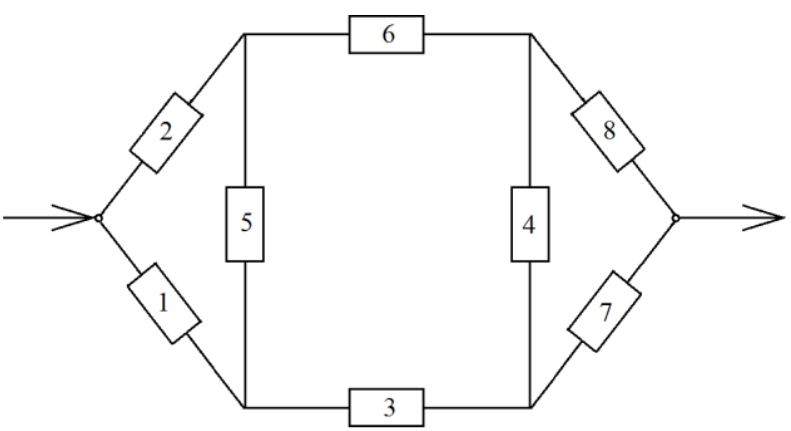

Fig. 2. Structural diagram of the aggregated subsystem.
In the figure 2 element 1 corresponds to the result of the aggregation of initial diagram elements $2,3,4,8$ while element 2 - to the results of the aggregation of elements 5, 6, 7, 9. Elements $3-8$ correspond to elements $10-15$ of the initial scheme. The reliability characteristics of the aggregated subsystem obtained are represented in Table 2 .

Table 3. Reliability characteristics of the merged subsystem's elements.

\begin{tabular}{|c|l|l|l|}
\hline Element & $T_{1}, \mathrm{yr}$. & $T_{0, \mathrm{yr} .}$ & $K_{a}$ \\
\hline 1 & 0.7051 & 0.02217 & 0.9695 \\
\hline 2 & 0.7924 & 0.01809 & 0.9777 \\
\hline 3 & 0.5000 & 0.03700 & 0.9311 \\
\hline 4 & 0.3600 & 0.01600 & 0.9574 \\
\hline 5 & 0.6000 & 0.02500 & 0.9600 \\
\hline 6 & 0.8000 & 0.01500 & 0.9816 \\
\hline 7 & 1.2000 & 0.001400 & 0.9988 \\
\hline 8 & 2.1000 & 0.001500 & 0.9993 \\
\hline
\end{tabular}

3. Applying the formulae (1) - (3) to the subsystem where the set $D_{1}$ contains 84 vectors, $D_{0}-172$ vectors one can obtain the next reliability characteristics of the subsystem $T_{1}=4,978 \mathrm{yr}$. , $T_{0}=0,01013 \mathrm{yr}$. , $K_{\mathrm{a}}=0,9980$. 4. Subsystem of parallel elements $17-18$ is merged to one element with $T_{1}=25,4911 \mathrm{yr}$. , $T_{0}=0,01120 \mathrm{yr}$. , $K_{\mathrm{a}}=$ 0,9996 .

Representing the initial system as consequential connection of two previously calculated subsystems with each other and with elements 1,16 and 19 of the initial system let us merge them to one element with reliability 
characteristics $T_{1}=0,3254$ yr., $T_{0}=0,02523$ yr., $K_{\mathrm{a}}=$ 0,9280 .

Thus, for the system considered

$$
T_{1}=0,3254 \mathrm{yr} ., T_{0}=0,02523 \mathrm{yr}, K_{\mathrm{\Gamma}}=0,9280 .
$$

The same result for of the system considered is obtained with the help of the structural functions method $[10,11]$.

\section{Conclusion}

The paper shows the possibilities of semi-Markov processes with common phase space of states application to model construction and operation analysis of energy systems using an example of independent SMP superposition. This approach lets to avoid of assumption on exponential distribution law and also take into account inspection, maintenance, etc. in the model of system. Unlike some other methods for finding the reliability and efficiency characteristics of energy systems, it allows to find not only probabilistic characteristics of the system, but also temporary ones. To solve the dimension problem one can use the algorithms of asymptotic and stationary phase merging of the systems [4-7].

The research was carried out within the state assignment of the Minobrnauki of Russia (№ 1.10513.2018/11.12), with financial support by RFBR (project № 18-0100392a).

\section{References}

1. Grabski F. Semi-Markov Processes Applications in System Reliability and Maintenance (Elsevier, 2015)

2. Obzherin Yu.E., Boyko E.G., Semi-Markov Models: Control of Restorable Systems with Latent Failures (Elsevier, Academic Press, 2015)

3. Janssen J., Manca R. Applied Semi-Markov Processes (Springer Science + Business Media, 2006)

4. Korolyuk V.S., Limnios N. Stochastic Systems in Merging Phase Space (World Scientific, Imperial College Press, 2005)

5. Silvestrov D., Silvestrov S. Nonlinearly Perturbed Semi-Markov Processes (Springer, 2017)

6. Koroluk V.S., Turbin A.F. Markovian Restoration Processes in the Problems of System Reliability (Naukova Dumka, Kiev, 1982)

7. Korlat A.N., Kuznetsov V.N., Novikov M.M., Turbin A.F., Semi-Markov Models of Recoverable Systems and Queuing Systems (Shtiinta, Chisinau, 1991)

8. Kashtanov V.A., Medvedev V.I. Theory of Complex Systems Reliability, $2^{\text {nd }}$ ed. (Fizmatlit, Moscow, 2010)

9. Rudenko Yu, N., Ushakov I.A. Reliability of Energy Systems, $2^{\text {nd }}$ ed (Nauka, Novosibirsk, 1989)

10. Beichelt F., Franken P. Reliability and Maintenance - Mathematical Methods. Radio i Svjas (Moscow, 1988)

11. Beichelt F., Tittmann P. Reliability and Maintenance - Networks and Systems (Mathematical Models. Chapman \& Hall, CRC Press, Boca Raton, London, New York, 2012) 